\title{
Understanding the Space-Time Continuum in Transformation of Cultural History by Analyzing ( $p=1)$ - Branes in Hyperspace
}

\author{
*Aakash Gupta, B.S
}

Saint Louis University

\begin{abstract}
Preface: Scientific dimensionality aids in determining phenomenon occurring in the cosmos in a way to relate time with the sequence of events. And, studying events in a three-dimensional Cartesian hyperspace provides a possibility to decode the pattern that has been followed by the laws and forces of nature, which made it possible to discover the history of the universe.

In this research paper I have tried to apply the dimensional analysis of String theory to the transformation of cultural history over monuments and paintings as some of the possible shapes human history has taken over the expanding period of time. The primary concept is based on conservation of energy in Physics, which I have related with an informational balance of historical past, hypothesizing that the historical details that have taken several forms can be analyzed individually in order to frame the ideal cultural history of mankind.
\end{abstract}

Furthermore, uncertainty principle has been kept in mind while comparing cultural history with p-branes analysis.

Keywords: Space-Time continuum: It is a theoretical mathematical model that combines space and time into a single interwoven continuum. Spacetime consists space relating to three-dimensions, and time as relating to one dimension, the 'fourth dimension'.

Hyperspace: It refers to the 4-dimensional spacetime of relativity, predicted by quantum mechanics.

Cultural History: It examines the records and narrative descriptions of past knowledge, customs, and arts of a group of people. Its subject matter encompasses the continuum of events occurring in succession leading from the past to the present and even into the future pertaining to a culture.

Understanding the space-time continuum in transformation of cultural history by analyzing (p=1)-branes in hyperspace

"Humankind has not woven the web of life. We are but one thread within it. Whatever we do to the web, we do to ourselves. All things are bound together. All things connect."

-Chief Seattle

Scientists and historians have been studying the notion of events associated to the past through the present in order to decode a pattern, which lies amongst things that seem incomparable. In order to continue this research, I thought of conducting a dimensional analysis, through scientific and historical perspectives, where all the events occur, rather than studying the events itself, and to understand the nature of the dimensions, which I call space and time.

Michio Kaku, theoretical physicist in string theory, claimed that the expansion of the universe could be explained through the dimensional analysis of information, which he referred to as quantum energy, and how it creates space with respect to time. ${ }^{1}$ His concept was based on Albert Einstein's fourth dimension, called space-time, which accounts for analyzing the physical phenomena in the cosmos.

The Grand Unified Theory, Einstein's incomplete work aiming at providing a fundamental description to the cosmological secrets, inspired lot of astrophysicists to give it a direction, yet with no absolute success. However, in 1925, Werner Heisenberg proposed a hypothetical claim that without space and time, it is difficult to formulate a physical theory, leading to the foundational assertion of string theory, which is considered one of the most acceptable theories by scientific communities to qualify for the unifying fundamental nature of forces.

String theory, as the name suggests, is based on strings, which are considered as the smallest quantum units expressing the thermodynamical nature of energy through space-time. However, in this research I will be focusing

\footnotetext{
* Corresponding Author: agupta21@slu.edu
} 
on p-branes, a type of string, which are physical objects that generalize the notion of a point particle to higher dimensions, where ' $p$ ' refers to the level of higher dimension of a brane, which ranges from zero to nine. In order to confine my research to space-time dimensional analysis, I will be considering $(p=1)$-branes, which includes Cartesian coordinates with an inclusion of fourth higher dimension, space-time, represented as $\mathrm{p}=1$.

Several theoretical ideas exist in the scientific community to provide a possible generalization about the expansion of the universe; however, string theory is the onethat explains it through the usage of dimensions. Performing dimensional analysis of information/energy to decode the history of the universe through $(\mathrm{p}=1)$-branes, provides a theoretical precision of Planck Length, which is $1.61619926 \times 10^{-35}$ meters. Physicists have now managed to quantify and relate variables of continuum through complex quantum equations, which gives a possibility to understand higher dimensions that are beyond our perception.

Applying the concepts of relativistic mechanics in string field theory, Dr. Kaku managed to find the proportionality between space and time coordinates in a complex phase plane, given as:

$\frac{\partial^{2} \chi^{\mu}(\sigma, \tau)}{\partial \tau^{2}}=c^{2} \frac{\partial^{2} \chi^{\mu}(\sigma, \tau)}{\partial \sigma^{2}}$

where, $\sigma$ and $\tau$ are the coordinates of space and time, respectively, on the string world sheet. The parameter $c^{2}$ defines the ratio of the string tension to the string mass per unit length as matter expands through the curvature of spacetime. $^{2}$

Analyzing these characteristic properties of strings and the rate of change of their coordinates in time, we can now generate a possible model for the history of our cosmos. In other words, these strings preserve the information from the cosmological past in the form of their characteristics and coordinates.

The conjecture of preserving information through string analysis does not violate the law of the conservation of energy and hence these equations can be further derived, on the similar basis of classical mechanics, to obtain the equations of rate of change of displacement, given as:

$\chi^{i}(\sigma, \tau)=x^{i}+\dot{x}^{i} \tau+i \sqrt{2 a^{\prime}} \sum_{n \neq 0}^{ \pm \infty} \frac{1}{n} a_{n}^{i}\left(\cos \frac{n \pi c \tau}{L}-i \sin \frac{n \pi c \tau}{L}\right) \cos \frac{n \pi \sigma}{L}$

The equation generates sinusoidal solutions (cosine and sine), and therefore claims that the strings vibrate in the wave pattern, having a frequency of $\frac{n \pi c \tau}{L} \mathrm{~Hz}$ and $\frac{1}{n} a_{n}^{i}$ amplitude.

These calculations aided in determining the total amount of matter that exist in the universe along with the possibility of anti-particles and dark matter to exist because of the complex term obtained in eq. 2.

Through the theoretically quantified hypothesis for the existence of opposite spinning particles existing in the nature, $\mathrm{S}$. Tosto gave an understanding of the expansion of the universe, claiming that the galaxies are found to be accelerating away from each other and therefore justifying the hypothesis obtained from $(\mathrm{p}=1)$ branes. ${ }^{3}$ According to the research conducted by P. Sharma, A. Tziolas, A. Wang, and C. Z. Wu on $(\mathrm{p}=1)$ branes, we can now determine the events that might have happened in the cosmological past by comparing the change in coordinates and entropy within a string to those instantaneous strings defining the current universe scenario. Thus, to understand the reverse motion of $\mathrm{p}$ strings in relatively negative time and compressed space, the equation can be deduced as

$S=-\frac{1}{4 \pi a^{\prime}} \int d \sigma d \tau \sqrt{h} h^{m n} \partial_{m} \chi^{\mu} \partial_{n} \chi_{\mu}$

which gives the possibility of having all the matter that exists today at an immensely dense point of infinite gravitational and anti-gravitational forces, which is responsible for the present we live in right now, that has formed structured orbital systems and astronomical objects as a result of the preservation of information that has been spreading over the curvature of space-time, and which is considered as the creation of the universe called the Big Bang. ${ }^{4}$

The two claims of string theory that everything in this universe follows a pattern and that information can never be lost, apply quite successfully in modeling the universe and reaching a logical and evidential concept of the Big Bang. However, I think it is not just limited within Astrophysics. The postulates of string theory are universally defined and can be applied in any field of research. Moreover, the co-founder of string field theory rightly said that "the string theory is the theory of everything". If we can discover the cosmological past through analyzing ( $\mathrm{p}=1)$ branes, I believe that there should be a way to deduce the history of humankind within this theory, as well, or that there might be a parallel between human history and the cosmological conclusions we have already obtained. 
Chronology has been consideredas the backbone of the study of history and has been shaped by one-dimensional linear model of history. ${ }^{6}$ The uni-dimensional concept of time restricts social scientists to perceive history in a confine manner, disregarding numerous possibilities, which might have existed. Also, it underlines the irreversibility of time. However, Prof. Jonathan Boyarin provides a theoretical multi-dimensional analysis of history, claiming that modern physics does challenge social scientists to change the notion of dimensionality in the field of contemporary history. His framework provides an urge to formulate a multi-dimensional model of history by merging the concept of space and time in analyzing social events that occurred in the past. ${ }^{7}$

Adding dimensionality to historical analysis, considering the concept of Big Bang as a model to use cultural history, provides several possibilities to explore our past by theoretically enabling the reversibility of time. In theory, space and time add the flexibility to move back and forth in time, since the information or the pattern of the events is encoded within dimensions. Understanding the notion of events occurring on the curvature of space relates time and thus provides a possibility to study the relationship between the two in multiple perspectives. However, my focus will be to study that relationship in order to analyze the transformation of historical information throughout time. Since travelling physically in time does not exist, therefore theoretical analysis of cultural history disregards the possibility of physical potential factors, aiding it towards formation of an ideal history. This can be understood as theoretically analyzing a body in motion, claiming that its total potential energy is being transformed to pure kinetic energy, ideally. However, in reality the situation is not the same. There are factors, such as force of friction, acceleration due to gravity, air resistance, and normal force, acting on the body in motion, which consume a relevant proportion of its total potential energy in overcoming these factors and thus preventing ideally determined energy transformations to occur.

The laws of nature claim that energy is never lost, although it transforms from one form to another. Therefore, on a similar basis I would like to coin a concept of informational balance, stating that information related to a specific event takes different forms with respect to time, but is always conserved within three-dimensional space.

Considering information balance in cultural history, events that occurred in the past took several shapes, monuments and paintings, is my areas of research, comprising the details of the event associated to the respective time. Analysis of those artifacts associated with a historical event might reveal the encoded reality of the historical past that a unidimensional model could not possibly deliver, however the possibility lies in filtering it through memory as how physicists managed to filter the history of our cosmos through characteristics and properties of higher dimensional p-branes of varying nature. Even though we have a scientifically accepted model to explain the origin of our universe, it is still an intelligent guess or can be considered as an ideal theoretical model of cosmological past comprising room for possible uncertainties.

Monumental description by Andreas Huyssen analyzes the transformation of spatial and temporal experience of human history into figurative architectures, which preserve our past in an architectural format. Those monuments serve as history itself that has transformed our selective memory in a specific format, entailing precise details about the historical past they are representing. Huyssen establishes that the Holocaust has been commodified in the global media society, as he says, "there is no pure space outside of commodity culture" (19). Memories of the Holocaust still preoccupy the world in several forms, one of which is Jewish Museum in Berlin, one of Huyssen's interests of space. Since time and space cannot be separated, the relationship between them mirrors the interconnected local and global of memory. Such spaces support as what he coins as the "culture of memory". Moreover, the environment created in such architectural pieces provides a real sense of feeling, aiding higher dimensions in realizing the past. ${ }^{8}$

On a similar basis, artists tried to create the environment through their paintings and preserve the historical past within art. The memories of events occurred takes the forms of paintings and drawings, which successfully express the goal and idea that the artists tried to convey. Picasso's Guernica(1937)serves as one of the best examples to qualify for conveying the tension and generating the environment in response to the bombing of Guernica during the Spanish Civil War, depicting the tragedies of war and the suffering that was inflicted upon individuals. This linkage between the incident and the way of representing its memory through an artwork, precisely justifies the concept of space and time. ${ }^{\dagger}$

There are academic institutions, which are coming forward to enhance the research of space and time in arts. One of these is the Finnish Academy of Fine Arts, providing research studies in Time and Space Arts, focusing on the interconnections between time, space, and the body, stating that " The student can place emphasis on one of the three orientation modules of the study programme: Moving Image, Site and Situation Art and Photography. The 
Therefore, the transformation of our historical past in the form of monuments and paintings justifies the concept of information balance, such that the details of our past take different forms and shapes with respect to time as the events occur in three-dimensional space. Moreover, the transformation of historical pasts doescomprise the existence of uncertainties. Within monuments, uncertainties lies in the form of empty spaces signifying that certain ideas or events cannot be expressed as a visual representation rather through spaces, which creates an environment, providing a sense of feeling, adding higher dimensions in realizing the past. Huyssen, in his interview at III Lisbon Summer School, urges social researchers and students to undertake cultural studies in monumental spaces, for a possibility that lies within empty spaces capable in revealing the memories of the past, which solidly perceptible architectures itself cannot unveil. Also, he emphasized the idea of multiplicity of meanings within arts and paintings, unlike texts and scriptures, serving as uncertainties within artworks. This claim justifies the concept of multiple rational perspectives, each of them possessing the capacity of a viable approach, which if combined together will lead to a possible ideal explanation of the respective artwork.

The interpretation of arts and designs decode reality through various logical approaches as Picasso rightly said, "If you give a meaning to certain things in my painting it may be very true, but it is not my idea to give this meaning. What ideas and conclusions you have got I obtained too, but instinctively, unconsciously. I make the painting for the painting. I paint the objects for what they are", ${ }^{9}$ which leaves a room for higher dimensional analysis and indicates the possibility of inaccuracy within transformations i.e. in relative perception and portrayal of events, supporting Huyssen's emphasison multimodal perception. Hence, if these details and information can be put together, it can lead us to an understanding of ideal historical pasts in the history of humankind.

After this research, I would say that the history of humankind is in no way different than the history of the universe.If physicists can hypothesize ideal Cosmological past through theoretical analysis of physically designed concepts of Kaku's field equations in the form of p-branes, I am sure Huyssen's multimodal perception holds a strong position that can lead us to the formation of ideal cultural history through critical analysis of information, which exists in different forms and shapes that renounced artists like Picasso has given themselves. All that needed is a paradigm shift in cultural history to go dimensionally richer in order to reach the absolute past of mankind. All the events, historical or physical, comprise characteristics, transformations, and progression in a similar manner; it is the pattern that distinguishes them and that is what needed to be decoded.

\section{REFERENCES}

[1] Kaku, M. (1994). Hyperspace: A scientific odyssey through parallel universes, time wraps, and the tenth dimension. Oxford University Press. ISBN: 0-385-47705-8. Retrieved from http://dzy428.webs.com/documents/Michio\%20Kaku\%20\%20 Hyperspace\%20(1994).pdf

[2] Purkayastha, A. D. (2013). Energy in the kantowski-sachs space-time teleparallel geometry. Pramana - Journal of Physics, 80(4), 571-581. doi: 10.1007/s12043-012-0492-1

[3] Tosto, S. (2013). Space-time uncertainty and cosmology: A proposed quantum model of the universe. Progress in Physics, 4, $1-23$.

[4] Sharma, P., Tziolas, A., Wang, A., \& Wu, Z. C. (2010). Spacetime singularities in string and its low dimensional effective theory. Int.J.Mod.Phys.A26, 273-300. doi: 10.1142/S0217751X11051408

[5] Belnap, N. (1992). Branching space-time. Springer, 92(3). Retrieved from http://www.jstor.org/stable/20117060

[6] Kern, S. (2003). The culture of time and space. Retrieved from http://monoskop.org/images/e/ea/Kern_Stephen_The_Culture _of_Time_and_Space_1880-1918_Chapters_1-4.pdf

[7] Boyarin, J. (1994). Space, time, and the politics of memory. Remapping memory: The politics of time-space. Retrieved from http://dx.doi.org/10.2307/2646349

[8] Huyssen, A. (2003). Present Pasts: Urban palimpsests and the politics of memory. Stanford, CA: Stanford University Press.

[9] Shabi, K (2013). Guernica meaning: Analysis and interpretation of painting by Pablo Picasso. LEGOMENON. Retrieved from http://legomenon.com/guernica-meaning-analysis-of-painting-by-pablo-picasso.html

objective of this study programme is to provide the students with a thorough understanding of these fields of art as well as insight to the manners in which they are interconnected and linked to the general art history".

Further details can be found at:

http://www.kuva.fi/en/studies/study-programs/time-and-space/

${ }^{*}$ https://www.youtube.com/watch?v=A8dRZFboang 